\title{
RESEARCH
}

Open Access

\section{Extracellular vesicles from human mesenchymal stem cells expedite chondrogenesis in 3D human degenerative disc cell cultures}

Daphne Hingert $^{1 *}$ D, Karin Ekström ${ }^{2,3}$, Jonathan Aldridge ${ }^{4}$, Rosella Crescitelli ${ }^{2,3}$ and Helena Brisby ${ }^{1,5}$

\begin{abstract}
Background: Extracellular vesicles (EVs) from human mesenchymal stem cells (hMSCs) are known to be mediators of intercellular communication and have been suggested as possible therapeutic agents in many diseases. Their potential use in intervertebral disc (IVD) degeneration associated with low back pain (LBP) is yet to be explored. Since LBP affects more than $85 \%$ of the western population resulting in high socioeconomic consequences, there is a demand for exploring new and possibly mini-invasive treatment alternatives. In this study, the effect of hMSCderived small EVs (sEVs) on degenerated disc cells (DCs) isolated from patients with degenerative discs and chronic LBP was investigated in a 3D in vitro model.
\end{abstract}

Methods: hMSCs were isolated from bone marrow aspirate, and EVs were isolated from conditioned media of the hMSCs by differential centrifugation and filtration. 3D pellet cultures of DCs were stimulated with the sEVs at $5 \times$ $10^{10}$ vesicles/ml concentration for 28 days and compared to control. The pellets were harvested at days 7, 14, and 28 and evaluated for cell proliferation, viability, ECM production, apoptotic activity, chondrogenesis, and cytokine secretions.

Results: The findings demonstrated that treatment with sEVs from hMSCs resulted in more than $50 \%$ increase in cell proliferation and decrease in cellular apoptosis in degenerated DCs from this patient group. ECM production was also observed as early as in day 7 and was more than three times higher in the sEV-treated DC pellets compared to control cultures. Further, sEV treatment suppressed secretion of MMP-1 in the DCs.

Conclusion: hMSC-derived sEVs improved cell viability and expedited chondrogenesis in DCs from degenerated IVDs. These findings open up for new tissue regeneration treatment strategies to be developed for degenerative disorders of the spine.

Keywords: Extracellular vesicles, Exosomes, Human mesenchymal stem cells, Intervertebral disc cells, Low back pain, Chondrogenesis

\footnotetext{
* Correspondence: daphne.hingert@gu.se

'Department of Orthopedics, Institute of Clinical Sciences, Sahlgrenska

Academy, University of Gothenburg, Gothenburg, Sweden

Full list of author information is available at the end of the article
}

\section{$\triangle B M C$}

(c) The Author(s). 2020 Open Access This article is licensed under a Creative Commons Attribution 4.0 International License, which permits use, sharing, adaptation, distribution and reproduction in any medium or format, as long as you give appropriate credit to the original author(s) and the source, provide a link to the Creative Commons licence, and indicate if changes were made. The images or other third party material in this article are included in the article's Creative Commons licence, unless indicated otherwise in a credit line to the material. If material is not included in the article's Creative Commons licence and your intended use is not permitted by statutory regulation or exceeds the permitted use, you will need to obtain permission directly from the copyright holder. To view a copy of this licence, visit http://creativecommons.org/licenses/by/4.0/ The Creative Commons Public Domain Dedication waiver (http://creativecommons.org/publicdomain/zero/1.0/) applies to the data made available in this article, unless otherwise stated in a credit line to the data. 


\section{Background}

Low back pain (LBP) affects more than $85 \%$ of the western population resulting in high socioeconomic consequences [1]. Chronic LBP is caused by multifactorial pathologies and further affected by psychosocial factors [2]. A certain interest has been directed towards intervertebral disc (IVD) degeneration, believed to be one of the major underlying pathologies [3]. It involves loss of proteoglycan and extracellular matrix (ECM) and increased expressions of pro-inflammatory cytokine and matrix metalloproteinases (MMPs), which affects the viability of the disc cells (DCs) in the IVDs $[4,5]$. Current treatment options are symptomatic and do not address the underlying degenerative process or promote regeneration. New treatment strategies with effective molecular agents that can be clinically applied in minimally invasive therapies are therefore warranted. Mesenchymal stem cell (MSC)-based therapies are of interest in tissue repair and regeneration due to their multilineage differentiation and immunomodulatory abilities [6]. One suggested strategy is injection of MSCs into degenerated IVDs and/or incorporation of growth factors to boost differentiation [7, 8]. However, growing evidence from studies on MSC effects on disc cells suggests that MSCs exert their therapeutic effects through paracrine signaling by secretion of bioactive factors and soluble peptides [9]. Extracellular vesicles (EVs) are secreted by the MSCs as mediators of intercellular communication, and they have shown a potential to drive regenerative processes in many diseases [10-12]. EVs have been reported to inhibit apoptosis in nuclear pulposus (NP) cells and suppress IVD degeneration in rat-tail models [13].

This study aimed to investigate the potency of small EVs (sEVs) isolated from hMSCs on degenerated DCs obtained from patients with degenerated IVDs and chronic LBP in a 3D in vitro model. The evaluation of the experiments was carried out in terms of cell proliferation, viability, ECM production, apoptotic activity, chondrogenesis, and cytokine secretions with the ambition that these findings, which are explicitly in connection with patients' cells, would pave ways for development of new regeneration strategies for treatments of spine degenerative disorders.

\section{Methods}

\section{Patients}

Intervertebral disc (IVD) tissues and bone marrow aspirates (BMAs) from iliac crest were collected from patients with chronic low back pain diagnosed with IVD degeneration undergoing spinal fusion surgery including removal of the intervertebral disc(s). The IVDs were graded according to the Pfirrmann grading system on magnetic resonance imaging as grade $3-4$. Disc tissues collected were obtained from the center of the IVD, aiming for NP tissue. However, due to disintegration of the IVD tissues, the collected tissues were considered a mix of NP and annulus fibrosus tissues. IVD tissues were obtained from 4 females and 1 male donors, age 34-50, while BMA was obtained from 1 female donor, age 40.

\section{Human MSC isolation and culture}

Human mesenchymal stem cells (hMSCs) were isolated and cultured as previously described in [8]. Briefly, BMA was collected in 3.2\% sodium citrate (Greiner Bio One, Kremsmuenster, Austria) after surgery and then centrifuged $(470 \times g, 20 \mathrm{~min})$. The mono-nuclear phase was collected and cultured in culture flasks (Corning, NY, USA) $\left(37^{\circ} \mathrm{C}\right.$ and $5 \% \mathrm{CO}_{2}$ ) in MSC media (DMEM-low glucose and $110 \mathrm{mg} / \mathrm{l}$ sodium pyruvate, with $4 \mathrm{mmol} / \mathrm{l} \mathrm{L}$-glutamine (Thermo Fisher Scientific, MA, USA) and 1\% penicillin/streptomycin, $4 \mathrm{ng} / \mathrm{ml}$ beta-fibroblast growth factor (FGF)) (Thermo Fisher Scientific) and 10\% human serum (HSE) (isolated in-house from donor's blood obtained from GeBlod, Gothenburg). Media were changed every 2 days, and the cells were passaged at $90 \%$ confluency.

\section{Disc cell isolation and culture}

Disc tissues were cut into small pieces and transferred to culture flasks (Corning, NY, USA) with an addition of 1 $\mathrm{mg} / \mathrm{ml}$ type II collagenase (Gibco Life, MA, USA). After a 24-h incubation $\left(37^{\circ} \mathrm{C}\right.$ and $\left.5 \% \mathrm{CO}_{2}\right)$, the cell suspension was centrifuged $\left(470 \times g, 4{ }^{\circ} \mathrm{C}, 5 \mathrm{~min}\right)$ and the cells were cultured $\left(37^{\circ} \mathrm{C}\right.$ and $\left.5 \% \quad \mathrm{CO}_{2}\right)$ in $\mathrm{DC}$ media (DMEM-low glucose with $110 \mathrm{mg} / \mathrm{l}$ sodium pyruvate, 4 $\mathrm{mmol} / \mathrm{l} \quad$ L-glutamine, $1 \%$ penicillin/streptomycin (Thermo Fisher Scientific), and 10\% HSE). The media were changed every 2 days, and the disc cells (DCs) were passaged at $90 \%$ confluency. All the DCs used in the study were in passage 5-6.

\section{Human MSC characterization}

The hMSCs were analyzed to confirm the expressions of CD73, CD90, and CD105 and the absence of CD11b, CD19, CD34, CD45, and HLA/DR using BD Stemflow hMSC analysis kit (BD Biosciences, CA, USA). The samples were prepared according to the manufacturer's instructions, and the data were acquired using a BD FACS Verse $^{\mathrm{TM}}$ instrument (BD Biosciences) and analyzed using FlowJo $^{\text {тм }} 10$ (Flow Jo, LLC, Ashland, OR, USA).

\section{Conditioned media collection and EV isolation}

hMSCs (approximately 10,000 cells $/ \mathrm{cm}^{2}$ ) were seeded in MSC media containing human serum for $24 \mathrm{~h}$ to allow cell attachment to the bottom of the flasks. The MSC media were removed, and the culture flask washed twice with phosphate buffer saline (PBS) prior to adding 
serum-free media (MSC NutriStem XF medium, Biological Industries, Kibbutz Beit-Haemek, Israel). Conditioned media $(\mathrm{CM})$ were collected every $48 \mathrm{~h}$, and the cells were passaged at $90 \%$ confluency. CM collected were centrifuged $\left(300 \times g, 4{ }^{\circ} \mathrm{C}, 5 \mathrm{~min}\right)$ and stored at $80^{\circ} \mathrm{C}$ until use. The hMSCs used in the study were in passage 4-7. The isolation of extracellular vesicles (EVs) was performed by a series of differential centrifugation and filtration of the $\mathrm{CM}$ as described in [14]. Briefly, $\mathrm{CM}$ were thawed on water bath and centrifuged at 16 , $500 \times g$ for $20 \mathrm{~min}$ followed by filtration through $0.22-\mu \mathrm{m}$ filters to deplete cell debris and large EVs. The sEV/exosomes were then pelleted by ultracentrifugation at 120 , $000 \times g$ for $70 \mathrm{~min}$ in a T-645.5 rotor (Sorvall $\mathrm{wx}$ Ultra series, Thermo Scientific, Rockford, IL, USA). The sEV pellets were re-suspended in cold PBS and stored at $80^{\circ} \mathrm{C}$ until use. The whole procedure was performed at $4{ }^{\circ} \mathrm{C}$.

\section{Characterization of EVs Nanoparticle tracking analysis}

The concentration and size distribution of the sEVs were determined by nanoparticle tracking (NTA). Briefly, the sEV samples were diluted $(200 \times$ and $1000 \times)$ with PBS and analyzed with Nanosight LM10/LM14 system (NanoSight Ltd., Malvern, UK) $(n=6)$. An automatic injection of the samples for each capture was carried out by a syringe pump, three 60 -s videos were taken for each dilution, and the analysis was carried out with Nanosight NTA 3.2 software. The concentration and size distribution were presented as the average concentration per cell \pm SEM and mean size \pm SEM in nanometer $(\mathrm{nm})$, respectively.

\section{Flow cytometry}

The hMSC sEVs were adhered to CD63-coated magnetic beads (Thermo Fisher Scientific, MA, USA) and analyzed for the presence of the tetraspanins CD9, CD63, and CD81 by flow cytometry according to the manufacturer's protocol with some small modifications. Briefly, for each sample, $10 \mu \mathrm{g}$ of sEVs was incubated with 100 , 000 beads overnight with gentle agitation. The bead-EVs were washed with $0.5 \% \mathrm{BSA}$ in PBS, incubated with CD9-APC-Vio-770, CD63-PE-Vio-770, and CD81PerCP-Vio700 or corresponding isotype control (Miltenyi Biotec, Bergisch Gladbach, Germany) for 40 min with gentle agitation at room temperature. The bead-EVs were washed three times and acquired on a FACSVerse (BD Bioscience, San Jose, CA, USA). The data was analyzed using FlowJo ${ }^{\text {tm }} 10$ (Flow Jo, LLC, Ashland, OR, USA).

\section{Transmission electron microscopy}

EV analysis by negative staining was performed as previously described [15]. Ten micrograms of vesicles was placed onto glow discharged 200-mesh formvar/carbon copper grids (Electron Microscopy Sciences, Hatfield Township, USA). After two washes in $\mathrm{H}_{2} \mathrm{O}$, sEVs were fixed in $2.5 \%$ glutaraldehyde. After two further washes in $\mathrm{H}_{2} \mathrm{O}$, the samples were stained with $2 \%$ uranyl acetate for $1.5 \mathrm{~min}$. Samples were examined on a digitized LEO 912AB Omega electron microscope (Carl Zeiss SMT, Oberkochen, Germany) at $120 \mathrm{kV}$ with a Veleta CCD camera (Olympus-SiS, Münster, Germany).

\section{Protein isolation and Western blot analysis}

Proteins from hMSCs were isolated using RIPA buffer (Thermo Fisher Scientific, Gothenburg, Sweden) and protein inhibitors (cOmplete ${ }^{\mathrm{Tm}}$, Mini Protease Inhibitor Cocktail, Roche, Basal, Switzerland). Small EVs isolated from hMSCs were mixed with RIPA buffer and used for the Western blot analysis. The protein concentrations were measured by Qubit (Thermo Fisher Scientific) according to the manufacturer's protocol. For Western blot analysis, $20 \mu \mathrm{g}$ of proteins was loaded, separated on precast 4-20\% polyacrylamide Mini-PROTEAN TGX gels (Bio-Rad Laboratories, Hercules, CA, USA), and transferred to PVDF membranes (Bio-Rad Laboratories, Hercules, CA, USA). The membranes were blocked with EveryBlot Blocking Buffer (Bio-Rad Laboratories, Hercules, CA, USA) and incubated with primary antibodies at $4{ }^{\circ} \mathrm{C}$ overnight. All primary antibodies were diluted in EveryBlot Blocking Buffer (Bio-Rad Laboratories). The primary antibodies used were Grp94 (1:1000 dilution, clone 9G10, Enzo Life Sciences, Solna, Sweden), anti-CD63 (1:1000 dilution, clone H5C6, BD Biosciences), anti-flotillin-1 (1:1000 dilution, clone EPR6041, Abcam, Cambridge, UK), and anti-Tom20 (1: 200 dilution, clone sc-136211, Santa Cruz Biotechnology, Santa Cruz, CA, USA). To investigate the CD63 expression, the separation was performed under non-reducing conditions. For the other proteins, the separation was performed under reducing conditions. The membranes were washed three times in $1 \times$ Tris-buffered salineTween (TBST) (Bio-Rad Laboratories) before incubation with the secondary antibody for $1 \mathrm{~h}$. The secondary antibody used for anti-flotillin-1 was anti-rabbit IgG (horseradish peroxidase conjugated, 1:5000 dilution, Harlan Sera-Lab, Loughborough, UK), the secondary antibody used for anti-CD63 and anti-Tom20 was anti-mouse IgG (horseradish peroxidase conjugated, 1:5000 dilution, Harlan Sera-Lab), and the secondary antibody used for anti-Grp94 was anti-rat IgG (horseradish peroxidase conjugated, 1:5000 dilution, Harlan Sera-Lab). All the secondary antibodies were diluted in EveryBlot Blocking Buffer (Bio-Rad Laboratories). After incubation with 
secondary antibodies, the membranes were washed three times in TBST and developed using the SuperSignal West Femto maximum sensitivity substrate (Thermo Fisher Scientific) and a ChemiDoc Imaging System (BioRad Laboratories).

\section{D pellet culture and $s E V$ treatment}

Approximately 200,000 DCs were placed in a polypropylene conical tube (Corning Inc., USA) with $0.5 \mathrm{ml}$ of chondrogenic media (DMEM-high glucose added with insulin, transferrin, and selenium (ITS-G; Thermo Fisher Scientific, MA, USA)), $5 \mathrm{mg} / \mathrm{ml}$ linoleic acid, $10 \mathrm{ng} / \mathrm{ml}$ transforming growth factor beta (TGF- $\beta 1$; R\&D Systems, MN, USA), $14 \mathrm{mg} / \mathrm{ml}$ ascorbic acid, $10^{-7} \mathrm{M}$ dexamethasone (Sigma-Aldrich, MS, USA), $1.0 \mathrm{mg} / \mathrm{ml}$ human serum albumin (Equitech-Bio Inc., KV, USA), and 1\% penicillin/streptomycin). The cells were centrifuged $\left(470 \times g\right.$ at $4{ }^{\circ} \mathrm{C}$ for $5 \mathrm{~min}$ ) and incubated $\left(37^{\circ} \mathrm{C}\right.$ and $5 \%$ $\mathrm{CO}_{2}$ ) for 3-4h to allow spheroid formation. For the EV treatment group, the media were replaced with $500 \mu \mathrm{l}$ of chondrogenic media containing sEVs $\left(5 \times 10^{10}\right.$ vesicles/ $\mathrm{ml})$. Chondrogenic media without the sEVs served as control. The media were replaced with fresh media with or without sEVs every $48 \mathrm{~h}$, the used media were collected and centrifuged $\left(300 \times g, 4{ }^{\circ} \mathrm{C}, 5 \mathrm{~min}\right)$, and the supernatants were stored at $-80^{\circ} \mathrm{C}$ for further analysis. The pellets were then harvested at days 7,14 , and 28 . Four replicates of pellets were cultured for each group and repeated with different donor cells $(n=5)$ separately. Two replicates were used for histology and two for biochemical analysis.

\section{Cell viability and LDH activity measurement Cell viability}

To each pellet, $50 \mu \mathrm{l}$ of cell counting kit 8 (CCK-8) solution (Dojindo, Munich, Germany) was added and incubated for $4 \mathrm{~h}\left(37^{\circ} \mathrm{C}\right.$ and $\left.5 \% \mathrm{CO}_{2}\right)$. The supernatant $(100 \mu \mathrm{l})$ was collected from each pellet in duplicates, and absorbance was measured at $450 \mathrm{~nm}$ by a microplate reader (BioTek, VT, USA). CCK-8 was performed at days 7,14 , and 28 before harvesting of the pellets.

\section{LDH activity}

Analysis of lactate dehydrogenase (LDH) was done on media collected from the different groups of DC cultures using lactate dehydrogenase (LDH) assay kit (Abcam) following the manufacturer's instruction. Absorbance was measured at $450 \mathrm{~nm}$ by a microplate reader (BioTek, VT, USA). The analysis was performed at days $4,7,13$, 22 , and 28. Technical duplicates were included in all the analyses and repeated with different donor cells $(n=5)$.

\section{Glycosaminoglycan and DNA assays}

DC pellets were solubilized with papain (1.5 mg papain/ $\mathrm{ml}$ (Sigma-Aldrich, MS, USA), $20 \mathrm{mM}$ sodium phosphate buffer, $1 \mathrm{mM}$ EDTA, and $2 \mathrm{mM}$ dithiothreitol) and incubated at $60^{\circ} \mathrm{C}$ overnight. The digested pellets were analyzed by glycosaminoglycan (GAG) and DNA assay kits (Chondrex, WA, USA). The assays were performed according to the manufacturer's instruction. Absorbance was read at $525 \mathrm{~nm}$ for GAG concentration, and fluorescence was read at excitation $360 \mathrm{~nm} / \mathrm{emission} 460 \mathrm{~nm}$ for DNA. Analyses were run in duplicates, and data presented as GAG content normalized to total DNA for each pellet.

\section{TUNEL assay}

Histology sections of DC pellets were deparaffinized, and TUNEL assay was carried out according to the manufacturer's guide (FragEL DNA Fragmentation Detection Kit, Fluorescent-TdT Enzyme, Merck Chemicals and Life Science AB, Sweden). Positive control was generated by submerging the section in $1 \mu \mathrm{g} / \mu \mathrm{l}$ DNase for $20 \mathrm{~min}$ prior to the other steps, and negative control was carried out by keeping the specimen in $1 \times$ buffer rather than in reaction mixture. Evaluation was performed with fluorescence microscopy (NIKON Eclipse E600, Japan), and NIS Elements software (Nikon Metrology NV, Europe) was used to perform fluorescent intensity quantification and determine the area of the pellets. The level of apoptosis was presented as pixels per square micrometer.

\section{Histological staining}

Harvested DC pellets were fixed with $4 \%$ formaldehyde (Histolab, Gothenburg, Sweden) and sectioned and stained with Alcian blue van Gieson, and the ECM components (proteoglycan and collagen) were evaluated under light microscopy (Nikon Eclipse E600).

\section{Immunohistochemistry PCNA, SOX9, KRT19, ACAN, COLIIA1}

Immunohistochemistry (IHC) was carried out to verify proliferation and characteristics of chondrocyte-like cells in the DCs. Briefly, paraffin-embedded sections were deparaffinized and rehydrated and antigen retrieval (Citrate buffer, $\mathrm{pH}-6,90^{\circ} \mathrm{C}$ for $20 \mathrm{~min}$ ) was carried out. Primary antibodies used include anti-PCNA (1:100, Abcam, Cambridge, USA), anti-Sox9 (1:1000, Abcam, Cambridge, USA), anti-KRT19 (1:100, Abcam, Cambridge, USA), anti-ACAN (1:500, Abcam, Cambridge, USA), and anti-COLIIA1 (1:100, Abcam, Cambridge, USA). After incubation at $4{ }^{\circ} \mathrm{C}$ overnight, blocking solution $(0.1 \%$ Triton X-100, 2\% BSA, and $100 \mathrm{mM}$ glycine in PBS) was added; for COLIIA1 sections, blocking with $3 \%$ BSA was used. Secondary antibodies include donkey anti-rabbit IgG Alexa Fluor 546 (1:200, Thermo Fisher 
Scientific, MA, USA) against SOX9, KRT19, PCNA, and ACAN, and goat anti-rabbit IgG Alexa Fluor 546 (1:200, Thermo Fisher Scientific, MA, USA) against COLIIA1. To enhance the detection of COLIIA1, the sections were incubated with SA-HRP (1:100, TSA Plus Cyanine 3 System kit, PerkinElmer, MA, USA) according to the manufacturer's guideline prior to nuclei counterstaining with ProLong ${ }^{\oplus}$ Gold Antifade Mountant (DAPI; Thermo Fisher Scientific, MA, USA). The samples were examined under fluorescence microscope (Nikon Eclipse E600, Japan), and NIS Elements software (Nikon Metrology NV, Europe) was used to determine the crosssectional area of the pellets and the number of positive cells and to quantify fluorescence intensity. The level of PCNA, SOX9, ACAN, and COLIIA1 expressions was presented as pixels/square micrometer, while for KRT19, 200 cells were counted and the immunopositive cells were presented as a percentage of the total count. The analysis of the images was conducted in duplicates for each group of pellet and was repeated with different donor cells $(n=5)$.

\section{Cell media/supernatant analysis Cytokine secretion}

Cytokine analysis was performed on cell-free supernatant collected from the DC pellet cultures (EV-treated or control, days $4,7,14,22$, and 28). The concentration of cytokines IL-1 $\beta$, IFN- $\alpha 2$, IFN- $\gamma$, TNF, CCL2, IL-6, CXCL8, IL-10, IL-12p70, IL-17A, IL-18, IL-23, and IL33 was measured using a bead-based immunoassay (LEGENDplex ${ }^{\mathrm{Tw}}$; Human Inflammation Panel 1, BioLegend, San Diego, USA) in accordance with the manufacturer's instructions. The samples were acquired on a FACS Verse (BD Bioscience, San Jose, CA) running FACSuite software (BD Bioscience). Data analysis was performed on FCAP Array software (Soft Flow Ltd., Pécs, Hungary).

\section{MMP-1 secretion}

The supernatant from pellet media was analyzed using human MMP1 ELISA Kit (Abcam, Cambridge, UK) following the manufacturer's instructions. Absorbance was measured at $450 \mathrm{~nm}$ by a microplate reader (BioTek, VT, USA).

All supernatant analyses were conducted with technical duplicates and were repeated with different donor cells $(n=5)$.

\section{Statistical analysis}

All statistical data are presented as mean \pm SEM. Data were analyzed via SPSS 25.0 software (IBM SPSS Statistics, NY, USA). Two-tailed Student's $t$ test was used to compare the means between two groups, and multivariate ANOVA with Tukey's post hoc was used for multiple comparison. $p<0.05$ was considered as statistically significant.

\section{Results \\ Characterization of hMSCs and sEVs confirmed their characteristics}

Human MSCs were isolated and expanded from bone marrow aspirates and further characterized using flow cytometry to confirm the mesenchymal lineage. The surface markers CD73, CD90, and CD105 of hMSCs were detected, and the hematopoietic lineage markers CD45, CD34, CD11b, CD19, and HLA-DR were absent (Fig. 1a) confirming the phenotypical characteristic of the isolated hMSCs. Extracellular vesicles (EVs) were isolated from the hMSC conditioned media (CM) using ultracentrifugation and analyzed by transmission electron microscopy (TEM), nanoparticle tracking analysis (NTA), flow cytometry, and Western blot to evaluate the integrity, size, concentration, and presence of EV markers (Fig. 1b-e). TEM pictures show cup-shaped elements, typical vesicle-like structures, with the size between 50 and $150 \mathrm{~nm}$ (Fig. 1b). NTA revealed that the size of sEVs ranges between 100 and $250 \mathrm{~nm}$ with the mean and mode size of $175 \pm 5.79 \mathrm{~nm}$ and $144 \pm 2.22 \mathrm{~nm}$, respectively (Fig. 1c). The number of sEVs secreted per hMSC was quantified to be $3.2 \pm 0.38 \times 10^{5}$. Western blot analysis showed that isolation of $\mathrm{sEVs}$ at two different batches, EV1 and EV2, expressed the typical exosome markers CD63 and flotillin-1 (Fig. 1d). The endoplasmic reticulum (ER) protein Grp94 and mitochondrial protein Tom 20 were only expressed in hMSC cellular proteins and not in the EVs, indicating no contamination of ER and mitochondria in the isolated EVs. Flow cytometry of EVs bound to CD63 beads shows that the tetraspanins CD9, CD63, and CD81 are detected on the membrane of the EVs (Fig. 1e). Collectively, these results indicate that we mainly isolated small EVs $(\mathrm{sEVs}<200 \mathrm{~nm})$, with the characteristics of exosomes.

\section{Small EVs promoted cell proliferation/viability and suppressed apoptosis in DCs}

In order to explore how hMSC-derived sEVs affect DCs' proliferation, CCK- 8 assay and immunohistochemistry for PCNA were carried out reflecting the viable cell number as well as the presence of proliferating cells in the DC pellets. The result from CCK- 8 revealed that DC pellets stimulated with SEVs showed a continuous cell proliferation from day 7 to day 28 with significantly higher number of viable cells $(p<0.01)$ compared to control at day 28. The number of viable cells further doubled from day 14 to day 28 in the EV-stimulated 
a

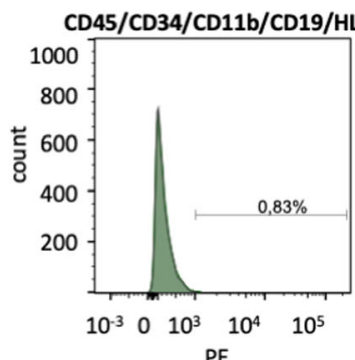

CD73

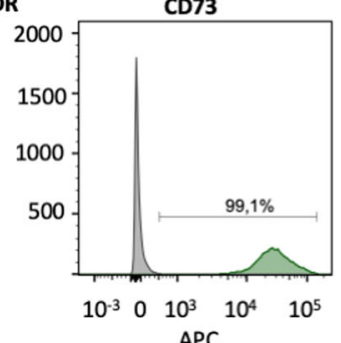

CD90
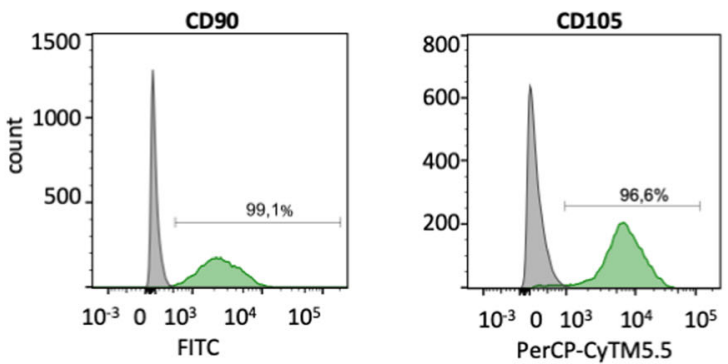

b

C
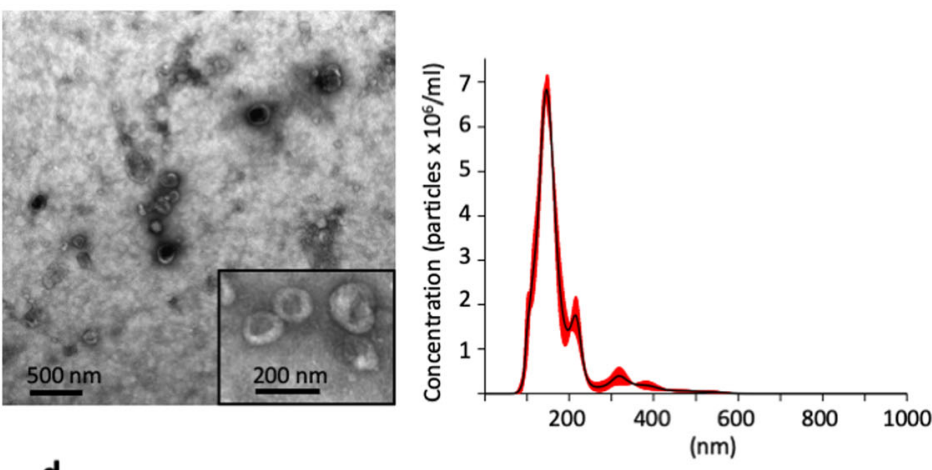

d
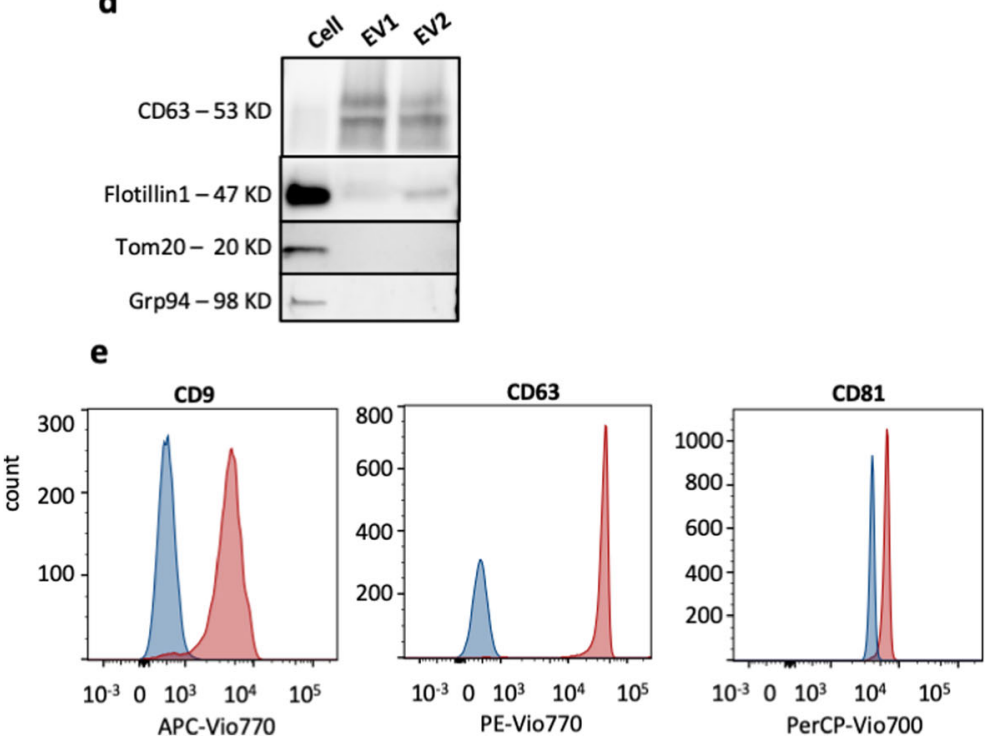

Fig. 1 (See legend on next page.) 
(See figure on previous page.)

Fig. 1 Characterization of hMSC-derived extracellular vesicles (EVs). a Bone marrow-derived hMSCs express the common MSC markers CD73, CD90, and CD105 and are negative for the hematopoietic markers CD45, CD340, CD11b, CD19, and HLA-DR (green curve, antibody stained sample; gray curve, isotype control). b Transmission electron microscopy (TEM) of sEVs. c Representative nanoparticle tracking analysis histogram shows the size distribution of sEVs. $\mathbf{d}$ Western blot analysis of two different isolations of hMSCs' sEVs (EV1 and EV2) and hMSCs (cell) shows that EVs contain the EV markers CD63 and flotillin-1 and are absent from the mitochondrial protein Tom-20 and ER protein Grp94. e Flow cytometry of sEVs bound to CD63 beads shows that sEVs are positive for the tetraspanins CD9, CD63, and CD81 (red curve, antibody stained sample; blue curve, isotype control)

group, while that of the control group remained the same (Fig. 2a). PCNA expression was detected in both EV-treated and non-treated pellets; however, in the EV group, a significantly higher level was exhibited compared to control (Fig. 2b, c), and the level was observed to follow a similar pattern to that of CCK-8 assay confirming increased cell proliferation with sEV stimulation.

LDH assay was used as an indicator of cell death, and TUNEL assay was performed additionally to investigate apoptosis in DC pellets. Lower LDH activity was detected in pellets treated with sEVs compared to control from day 4 to day 14 indicating low cell death with sEV treatment; however, the level raised at day 22 and declined slightly at day 28 (Fig. 2d). TUNEL assay revealed the presence of cells with fragmented DNA in both groups (Fig. 2e, f); however, at all time points, significantly lower level was detected in sEV-treated pellets compared to control with an increasing level observed from day 7 to 28 in the control group. This result conforms to that of LDH activity validating that stimulation with sEVs suppressed cell death in DC pellets.

\section{Small EVs induced early chondrogenesis in DCs from degenerated IVDs}

To validate the characteristic of NP/chondrocyte-like cells in DCs isolated and to confirm the occurrence of chondrogenesis, the presence of KRT19 and SOX9 was investigated by IHC as KRT19 is one of the markers of NP cells [16] and SOX9 is known as the early indicator of chondrogenesis [17]. The expression of KRT19 was observed in all the pellets confirming the chondrocytelike cell characteristics of the DCs isolated (Fig. 3a, b). SOX9 expression was detected throughout the time points; however, significantly higher level was detected in pellets stimulated with sEVs at days 7 and 14 compared to control (Fig. 3c, d). At the latest time point, 28 days, SOX9 expression was increased in the control compared to the EV-treated group. This implied that chondrogenesis took place earlier when treated with sEVs compared to control.

\section{Small EVs enhanced early production of ECM}

To reverse IVD degeneration, it is vital to influence the DCs in degenerated IVDs to become viable and to produce ECM in order to preserve the integrity of the discs.
In an attempt to investigate if the sEVs from hMSCs could influence the DCs to produce vital ECM, GAG assay, Alcian Blue van Gieson (ABvG) histology staining, and $\mathrm{IHC}$ were carried out to investigate the production of GAG, collagen type II (COLIIA1), and aggrecan (ACAN) in DC pellets from day 7 to 28 .

GAG assay showed an early production of GAG after EV treatment, with significantly higher levels in DCs treated with sEVs compared to control at days 7 and 14 $(p<0.01)$. However, a continuous increase in GAG production was observed in the control group from day 7 to 28, while in the EV group, a significantly high production was observed from day 7 to day 14 where the pellets yielded highest production already at day 14 (Fig. 4a). This is supported by the results from ABvG staining as DCs treated with sEVs showed high proteoglycan accumulation as early as in day 7 and throughout the culture period (Fig. 4b), confirming that sEVs from hMSCs promoted an increased and early production of ECM in degenerated DCs.

COLIIA1 and ACAN are some of the main ECM compositions of the nucleus pulposus; in fact, COLIIA1 is also known as the late indicator of chondrogenesis $[18,19]$. In DC pellets treated with sEVs, high COLIIA1 expression was observed as early as in day 7 and the level was significantly higher than control and remained similar throughout the experiment (Fig. 5a, b). The level of COLIIA1 and ACAN remained at the same expression level throughout the experiment in the EV group. Furthermore, the level of ACAN expression after sEV treatment was significantly higher than control in days 14 and 28 (Fig. 5c, d). This finding is consistent with that of GAG assay, and it gives affirmation that sEV treatment expedited and enhanced ECM production in DC pellets.

\section{Secreted cytokines and MMP-1 levels in cell media}

In order to study the secretory response of DCs after exposure to the hMSCs' sEVs, the concentration of different cytokines (IL-1 $\beta$, IFN- $\alpha 2$, IFN- $\gamma$, TNF, CCL2, IL-6, CXCL8, IL-10, IL-12p70, IL-17A, IL-18, IL-23, and IL33) and MMP-1 was measured. However, only monocyte chemoattractant protein 1 (MCP-1), interleukin 6 (IL-6), and interleukin 8 (IL-8) were detected in the media. The level of IL- 6 and IL-8 in the medium from EV-treated 
a
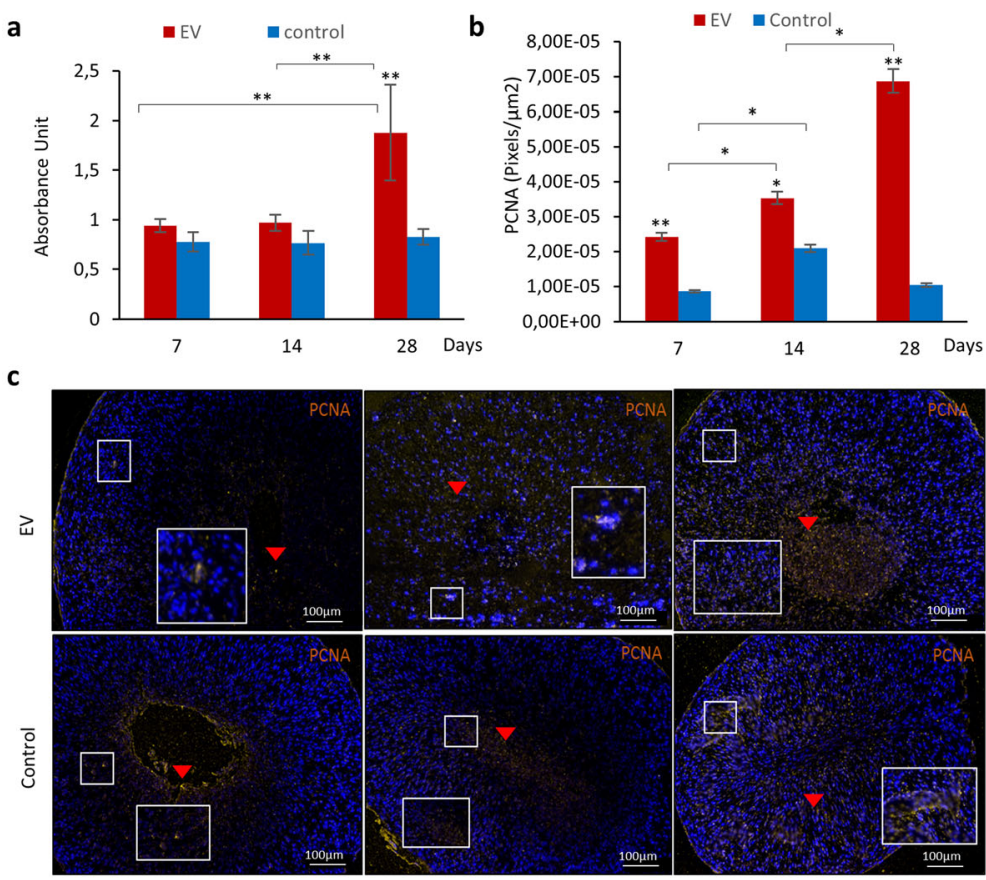

Day 7

d

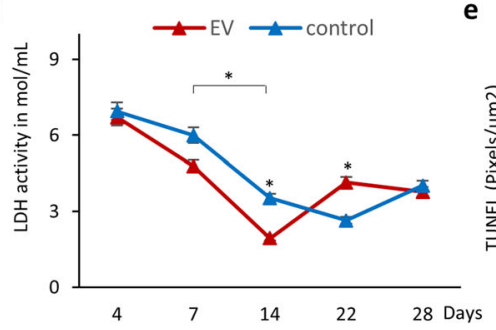

Day 14

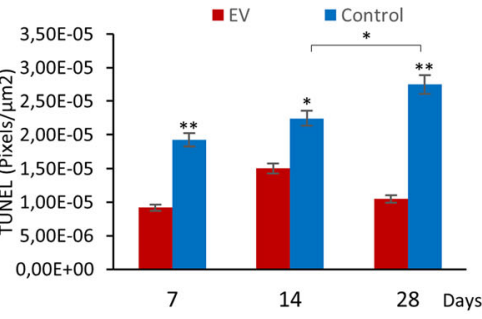

f

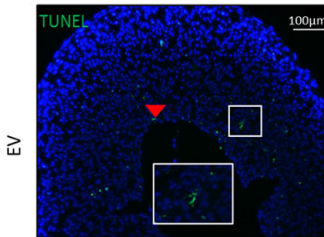

10um TUNEL $\quad$ 100um
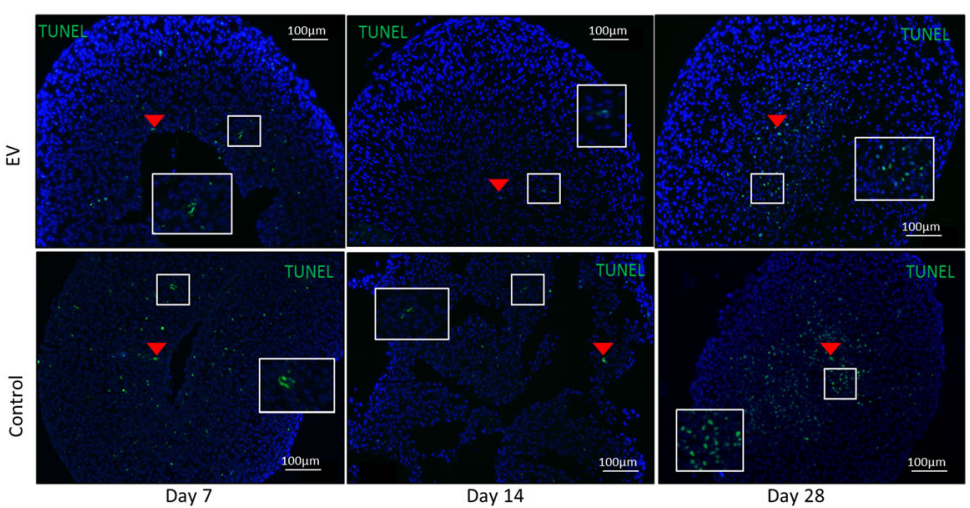

Fig. 2 Cell proliferation and expression of cell proliferation markers after EV treatment. a lllustration of cell proliferation from day 7 to 28 in DC pellets treated with EVs compared to control. The result is presented in the unit of absorbance. $\mathbf{b}$ The bar graph presents the quantitative level of PCNA-positive cells in histological sections of DCs treated with sEVs compared to control from day 7 to 28 . The results are presented as pixels/ square micrometer. c Representative images of the expressions of PCNA in DC pellets treated with sEVs from day 7 to 28 . Yellow colors indicated by red arrows are the positive expressions of PCNA, while green colors are that of apoptotic cells in the pellets. Blue dots are the nuclei of DCs. $\mathbf{d}$ LDH activity of DC pellets. The line graph illustrates LDH activity (cell death) in DC pellets from day 7 to 28. e The bar graph presents the quantitative level of TUNEL assay in histological sections of DCs treated with sEVs compared to control from day 7 to 28 . $\mathbf{f}$ Representative images of the expressions of apoptotic cells in DC pellets treated with sEVs from day 7 to 28 , respectively. Values are the mean \pm SEM $(n=5){ }^{*} p<0.05,{ }^{* *} p<0.01$ 

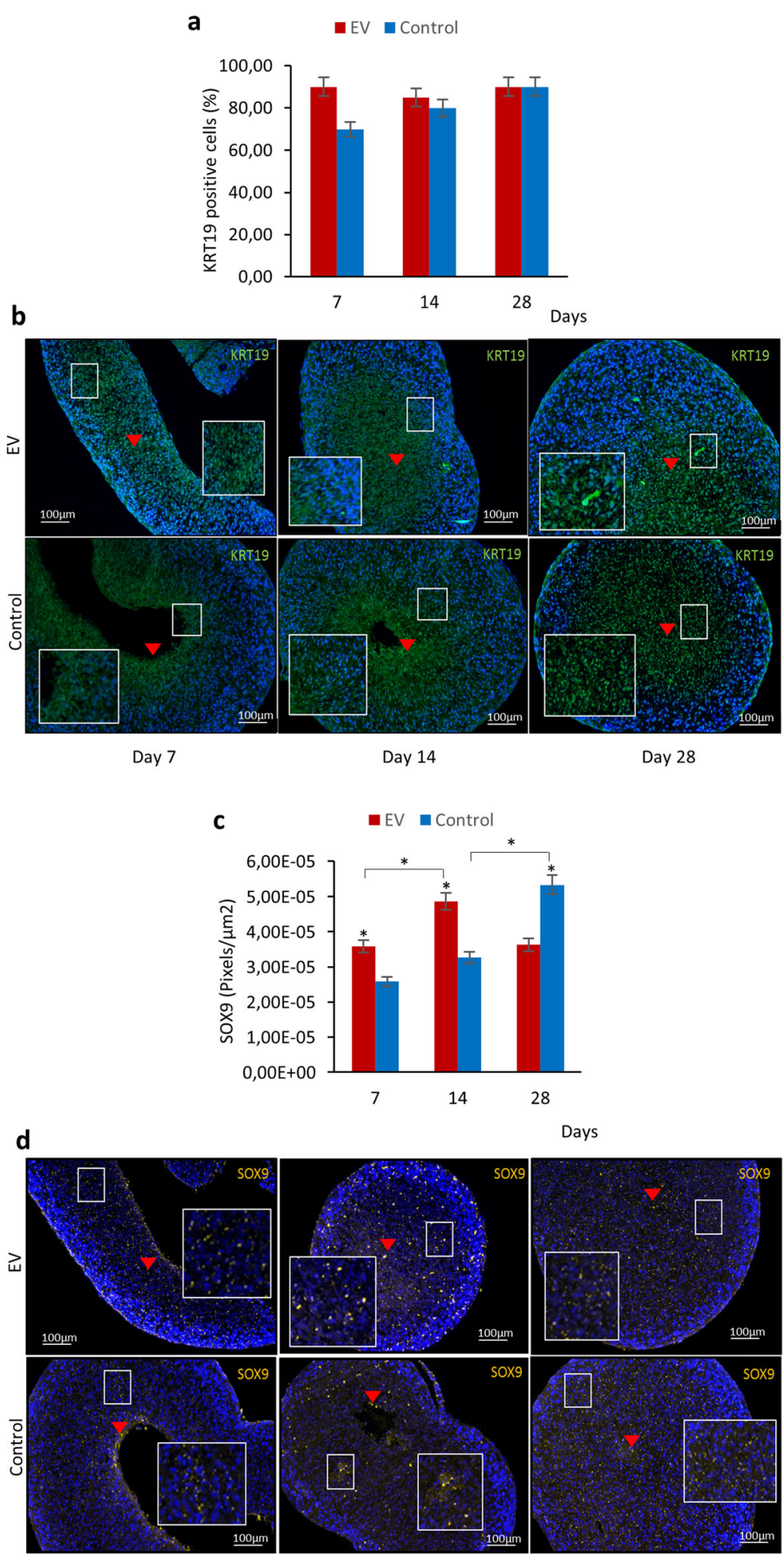

Fig. 3 Chondrocyte-like cell characteristics of DCs isolated from degenerated IVDs after hMSCs' sEV treatment. a The bar graph presents the percentage of KRT19-positive cells in histological sections of DCs treated with sEVs compared to control from day 7 to 28. b Representative images showing the expressions of KRT19 in DC pellets treated with sEVs from day 7 to 28. c The bar graph illustrates the quantitative level of SOX9-positive cells in histological sections of DC pellets for the same time points. $\mathbf{d}$ Representative images showing the expression of SOX9 in DC pellets treated with sEVs from day 7 to 28 . Quantitative results in $\mathbf{a}$ and $\mathbf{c}$ are presented as pixels/square micrometer while green colors in $\mathbf{b}$ and $\mathbf{d}$ indicated by red arrows are the positive expressions of KRT19, while yellow colors are that of SOX9 in the pellets, respectively. Blue dots are the nuclei of DCs. Data represents mean \pm SEM $(n=5)$. * $p<0.05$ 
a

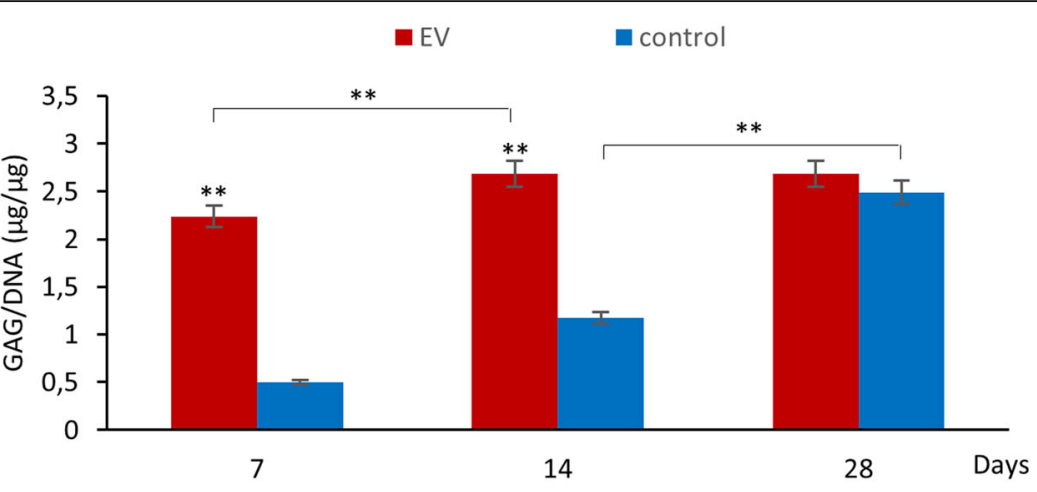

b

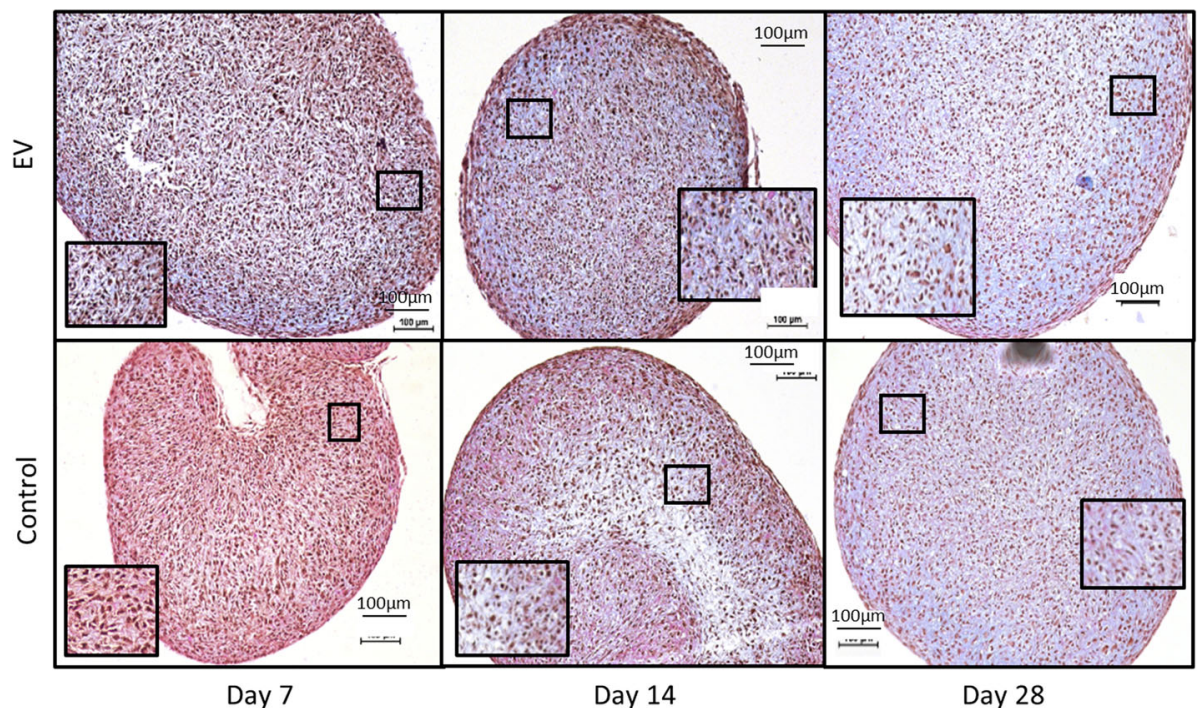

Fig. 4 GAG production and histological staining of DC pellets treated with sEVs from day 7 to 28. a Quantification of GAG production in DC pellets. Values presented in bar charts are the mean \pm SEM $(n=5) .^{*} p<0.05$ and ${ }^{* *} p<0.01$. b Alcian Blue van Gieson staining of DC pellets. Histology sections present proteoglycan (blue) and collagen (pink), and black dots represent nuclei of the cells

pellets was highest at day 7 and decreased at later time points. However, no significant differences between EVtreated pellets and control were detected for any of the cytokines (Fig. 6a-c). The release of MMP-1, which is a collagenase responsible for degradation of collagens in the IVDs during IVD degeneration [20], decreased over time in both EV-treated and control (Fig. 6d). Interestingly, DCs treated with hMSCs' sEVs released a lower level of MMP-1 compared to control.

\section{Discussion}

Cell therapy approaches such as injection of MSCs have been recognized as a promising treatment strategy for LBP caused by IVD degeneration since MSCs can differentiate into chondrocyte-like cells and promote cell proliferation [21] and inhibit apoptosis [13]. However, several studies have provided compelling evidence indicating that the therapeutic effects of MSCs are mainly mediated by paracrine mechanisms, particularly the secretion of EVs [10, 12, 22, 23]. Therefore, the use of hMSC-derived EVs may serve as a more appropriate alternative for treatment of IVD degeneration. Unlike hMSCs, EVs are more stable and not easily influenced by the inflammatory microenvironment in the degenerated IVDs [24]. EVs may therefore be used to promote IVD repair by combating destructive effects of proinflammatory cytokines and MMPs in the disc microenvironment.

In this study, it is demonstrated in a 3D in vitro model that hMSC-derived sEVs may fulfill these requirements. The results revealed that sEVs can improve cell viability and proliferation and expedite chondrogenesis in DCs from degenerated IVDs of patients suffering severe LBP. EV treatment induced early production of crucial ECM components such as proteoglycan, aggrecan, and collagen type II while suppressing apoptosis and the secretion of MMP-1, a process that is essential for proper IVD 


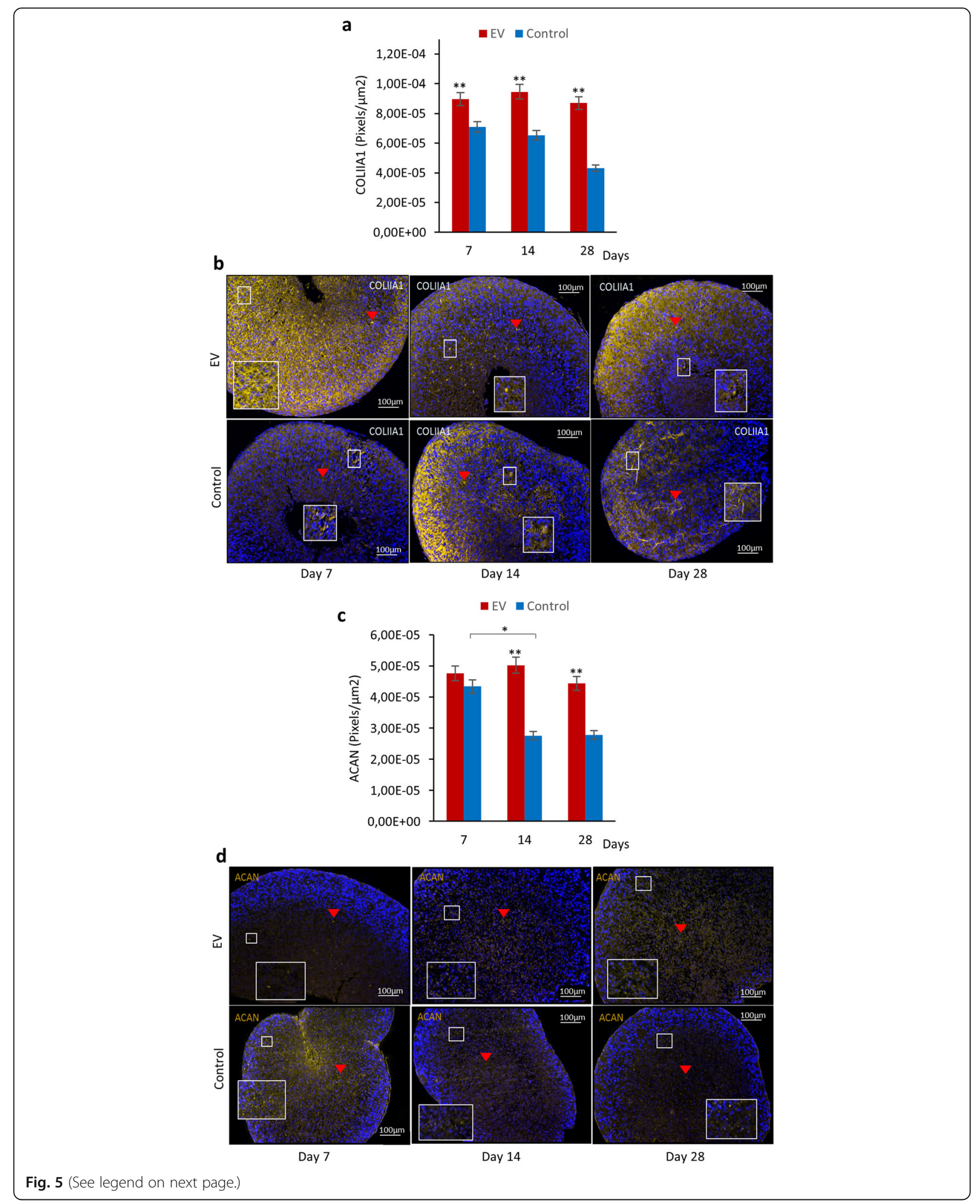


(See figure on previous page.)

Fig. 5 Expressions of collagen type II (COLIIA1) and aggrecan (ACAN) in DC pellets over a 28-day period. a The bar graphs illustrate the level of COLIIA1-positive cells in DC pellets. b Representative images showing the expressions of COLIIA1 in DC pellets treated with sEVs from day 7 to 28. c The bar graphs illustrate the level of ACAN-positive cells in DC pellets. $\mathbf{d}$ Representative images showing the expressions of ACAN in DC pellets treated with sEVs from day 7 to 28 . Quantitative results in $\mathbf{a}$ and $\mathbf{c}$ are presented as pixels/square micrometer while yellow colors indicated by red arrows in $\mathbf{b}$ and $\mathbf{d}$ are positive expressions of the proteins in the pellets. Blue dots are the nuclei of DCs. Data represents mean \pm SEM ( $n=$ 5). ${ }^{*} p<0.05$ and ${ }^{* *} p<0.01$

regeneration. Small EVs also increased viability and proliferation of DCs.

The level of proliferation of DCs in pellet culture over a 28-day period was previously reported to be consistent throughout the culture period and therefore speculated that it was because these DCs were mature cells exposed to degenerative environment and were hard to influence [8]. However, with SEV treatment, a continuous increase in the level of proliferating cells was detected from day 7 to 28 , which is in line with previous report where
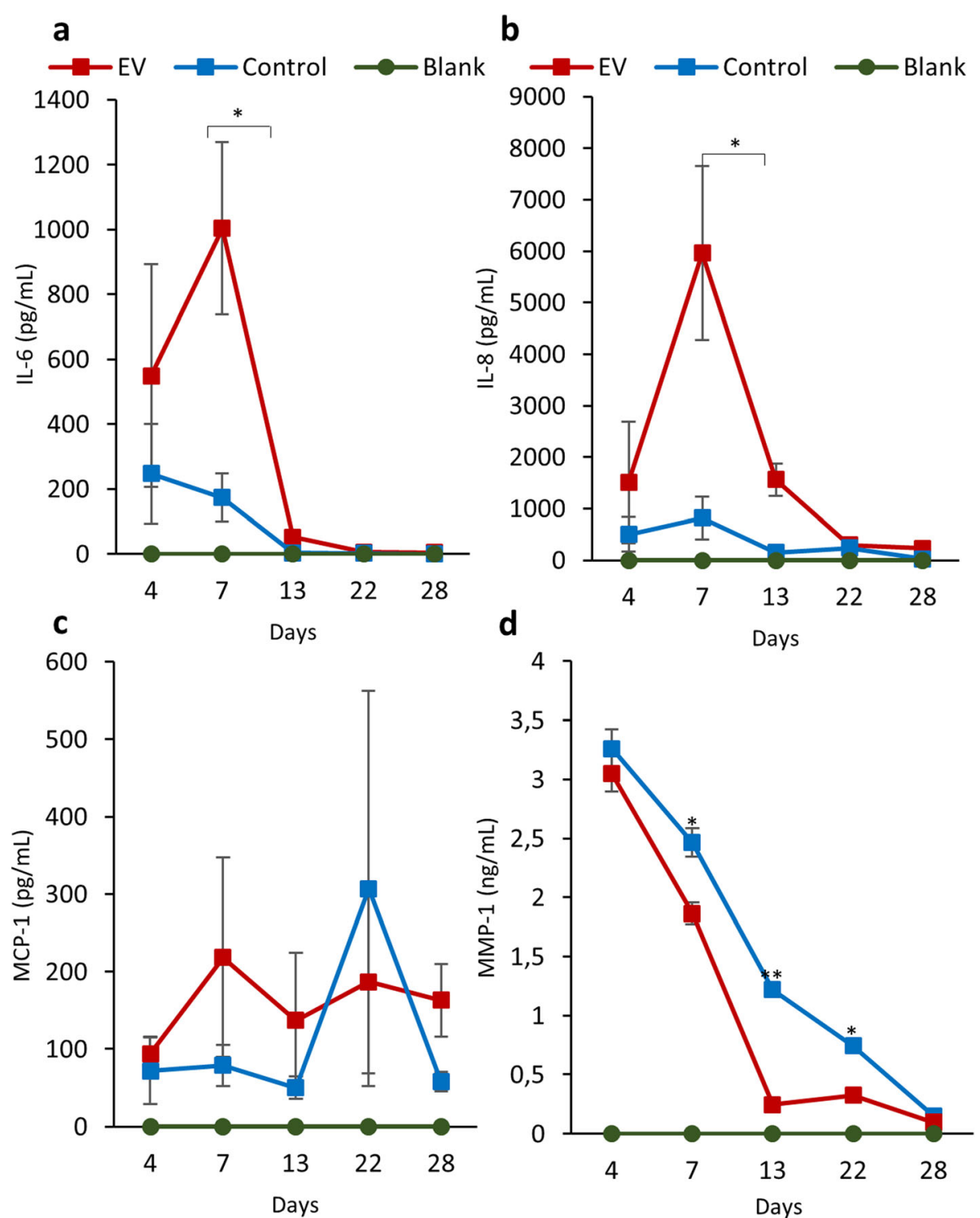

Fig. 6 The secretion of cytokines and MMP-1 by the DCs after sEV treatment. a-c The line graphs illustrate the concentration of cytokines IL-6, IL8, and MCP-1 respectively, secreted by the DCs over a 28-day period. $\mathbf{d}$ The line graph represents the level of MMP-1 secreted by DCs treated with sEVs compared to control. Data represents mean $\pm \operatorname{SEM}(n=5) .{ }^{*} p<0.05$. Fresh media without the sEVs served as blanks 
exosomes promoted cell proliferation of nucleus pulposus (NP) cells through cellular uptake of the vesicles [25]. These findings show that proliferation of mature DCs can be promoted given the right stimulus.

Previous studies have reported that exosomes isolated from MSCs inhibit apoptosis in cartilage cells [11, 13] and ameliorate endoplasmic reticulum stress-induced apoptosis by activating AKT and ERK signaling in a rattail model [26]. In this study, reduced LDH activity and apoptosis were observed in DC pellets stimulated with sEVs indicating that sEVs could be the key bioactive factors for preventing DC's apoptosis in the degenerated IVDs.

The presence of SOX9 and KRT19 expressions $[18,19]$ also confirmed the chondrocyte-like cell characteristic of the DCs isolated. Another characteristic of chondrocytelike cells is the ability to produce ECM [27]. The main compositions of IVDs are collagen and proteoglycans forming a strong framework that holds the cells in the ECM where matrix degrading enzymes (MMPs) and their inhibitors are kept in homeostasis. As IVD degeneration progresses, the concentration of pro-inflammatory factors along with MMPs increases while the concentration of MMP inhibitors decreases, resulting in an increased matrix degradation $[27,28]$. This threatens the microenvironment of the cells as they depend on the ECM for survival [2]. In DCs from degenerated IVDs, stimulation with SEVs induced an earlier and a significantly higher proteoglycan accumulation, GAG production, and expressions of COLIIA1 and ACAN compared to the control. This increased production of ECM may account for the suppressed MMP-1 production by the DCs as the ECM provided the DCs with favorable environment. Exosomes derived from hMSCs were also reported to suppress $\mathrm{H}_{2} \mathrm{O}_{2}$-induced NP cell inflammation [29]. This means that sEVs were able to assist the DCs to rebalance homeostasis in this 3D model. This was further supported by the decreased concentration of MMP-1 in the media in the EV group.

Previous findings report promising results when stimulating DCs with growth factors such TGF-beta and BMP3 and also with conditioned media derived from hMSCs $[8,30,31]$ as these signaling molecules are believed to act either on themselves (autocrine) or on neighboring cells (paracrine) [32, 33] to bring about the observed regenerative effects. In this study, it was demonstrated that with EV stimulation, an enhanced ECM production was observed as early as in day 7 in these $3 \mathrm{D}$ pellet culture models. It is of interest to understand which molecules are responsible for these effects; however, in this study, the content of the hMSCs' sEVs was not analyzed in depth. On the other hand, small interdonor variation was detected, and this could be due to the fact that the disc tissues used in the study were at similar levels of degeneration. It could also be speculated that the concentration of EVs used in this study had a strong impact on the degenerated disc cells as this concentration was slightly higher than previously used concentration [34]. The rationale behind using this slightly higher concentration was on the grounds that a 3D model was used for this study and to ensure that enough EVs were present to influence the cells.

EVs from hMSCs have previously been shown to contain a wide range of different growth factors, cytokines, and RNA [35]. It is reasonable to speculate that a mixture of these factors is responsible for causing the effects in the DCs. It could be speculated that the SEVs either were internalized by the DCs [13] for intercellular communication or were delivering bioactive molecules involved in direct cell stimulation [36].

EVs are further known for their involvement in cytokine transportation [37]. Both IL-6 and IL-8 have been found to be associated with secretory vesicles. Upon stimulation with IL-1, mast cells have been reported to release IL-6 containing vesicles [38] while IL-8 has been shown to be transported in the sEVs as inflammatory mediators $[39,40]$. The higher levels of IL-6 and IL-8 detected in the media in the EV group compared to control at day 7 are most likely caused by DC secretion after sEV stimulation. However, we cannot rule out that the detected IL- 6 and IL- 8 are soluble and not bound to the EV membrane.

\section{Conclusion}

Human MSC-derived sEVs may have a potential for therapeutic applications in patients with LBP and disc degeneration, as they mimic several positive biological actions of hMSCs. It may also limit the issues of cell maldifferentiation or mutations and cell handling concerning cell therapy. Thus, the findings from this study open up treatment strategies where sEVs can be engineered to express and deliver molecules that can direct target cells towards regeneration.

\section{Abbreviations \\ ACAN: Aggrecan; ABvG: Alcian blue van Gieson; BMA: Bone marrow aspirate; COLIIA1: Collagen type II; DCs: Disc cells; DNA: Deoxyribonucleic acid; \\ ECM: Extracellular matrix; FGF: Beta fibroblast growth factor; \\ GAG: Glycosaminoglycan; hMSCs: Human mesenchymal stem cells; \\ HSE: Human serum; IL-6: Interleukin-6; IL-8: Interleukin-8; IVD: Intervertebral disc; LDH: Lactate dehydrogenase; LBP: Low back pain; MSCs: Mesenchymal stem cells; MMP-1: Metalloproteinase-1; NTA: Nanoparticle tracking analysis; NP: Nucleus pulposus; sEVs: Small extracellular vesicles; WB: Western blotting}

\section{Acknowledgements}

This study was supported by the funding from the Swedish state under the agreement between the Swedish government and the country councils, the Alf-agreement, the Hjalmar Svensson foundation, and the Dr. Felix Neubergh foundation.

Data availability

Not applicable 


\section{Authors' contributions}

D.H: conception and design, conducted most of the experiments, data analysis and interpretation of the results, and manuscript writing; K.E: conception and design, carried out some of the experiments, interpretation of the results, and manuscript writing; J.A: performed some of the experiment, and data analysis; R.C: performed some of the experiments; H.B: conception and design, collected patients' samples, interpreted the results, manuscript writing, and financial support. All the authors have read and approved the manuscript.

\section{Funding}

The study was financed by grants from the Swedish state under the agreement between the Swedish government and the country councils, the Alf-agreement, and the Dr. Felix Neubergh foundation. Open access funding provided by University of Gothenburg.

\section{Availability of data and materials}

All data generated or analyzed during this study are included in this article.

\section{Ethics approval and consent to participate}

All human tissues/cells were collected with ethical permission approved by the Regional Ethical Review Board at Sahlgrenska Academy, Gothenburg University, Gothenburg, Sweden (Dnr 532-04), and with informed consent from all patients

\section{Consent for publication}

Informed consent for publication has been received.

\section{Competing interests}

The authors declare that they have no competing interests.

\section{Author details}

'Department of Orthopedics, Institute of Clinical Sciences, Sahlgrenska Academy, University of Gothenburg, Gothenburg, Sweden. ${ }^{2}$ Sahlgrenska Cancer Center, Department of Surgery, Institute of Clinical Sciences, Sahlgrenska Academy, University of Gothenburg, Gothenburg, Sweden. ${ }^{3}$ Wallenberg Centre for Molecular and Translational Medicine, University of Gothenburg, Gothenburg, Sweden. ${ }^{4}$ Department of Rheumatology and Inflammation Research, Institute of Medicine, University of Gothenburg, Gothenburg, Sweden. ${ }^{5}$ Department of Orthopedics, Sahlgrenska University Hospital, Gothenburg, Sweden.

\section{Received: 23 April 2020 Revised: 2 July 2020}

Accepted: 14 July 2020 Published online: 29 July 2020

\section{References}

1. van Hooff ML, et al. The Nijmegen decision tool for chronic low back pain. Development of a clinical decision tool for secondary or tertiary spine care specialists. PLoS One. 2014;9(8):e104226.

2. Pearce RH, Grimmer BJ, Adams ME. Degeneration and the chemical composition of the human lumbar intervertebral disc. J Orthop Res. 1987; 5(2):198-205.

3. Ekman M, et al. Burden of illness of chronic low back pain in Sweden: a cross-sectional, retrospective study in primary care setting. Spine (Phila Pa 1976). 2005;30(15):1777-85.

4. Ehlicke $F$, et al. Intervertebral disc regeneration: influence of growth factors on differentiation of human mesenchymal stem cells (hMSC). Int J Artif Organs. 2010;33(4):244-52.

5. Hingert D, et al. Pathological effects of cortisol on intervertebral disc cells and mesenchymal stem cells from lower back pain patients. Cells Tissues Organs. 2019:207(1):34-45.

6. Squillaro T, Peluso G, Galderisi U. Clinical trials with mesenchymal stem cells: an update. Cell Transplant. 2016;25(5):829-48.

7. Henriksson HB, et al. Transplantation of human mesenchymal stems cells into intervertebral discs in a xenogeneic porcine model. Spine (Phila Pa 1976). 2009:34(2):141-8.

8. Hingert D, et al. BMP-3 promotes matrix production in cocultured stem cells and disc cells from low back pain patients. Tissue Eng Part A. 2020;26(1-2): 47-56. https://doi.org/10.1089/ten.TEA.2019.0125.
9. von Bahr $L$, et al. Analysis of tissues following mesenchymal stromal cell therapy in humans indicates limited long-term engraftment and no ectopic tissue formation. Stem Cells. 2012;30(7):1575-8.

10. Cosenza S, et al. Mesenchymal stem cells derived exosomes and microparticles protect cartilage and bone from degradation in osteoarthritis. Sci Rep. 2017;7(1):16214.

11. Zhang $\mathrm{S}$, et al. MSC exosomes mediate cartilage repair by enhancing proliferation, attenuating apoptosis and modulating immune reactivity. Biomaterials. 2018;156:16-27.

12. Nakamura $Y$, et al. Mesenchymal-stem-cell-derived exosomes accelerate skeletal muscle regeneration. FEBS Lett. 2015;589(11):1257-65.

13. Cheng $X$, et al. Mesenchymal stem cells deliver exogenous miR-21 via exosomes to inhibit nucleus pulposus cell apoptosis and reduce intervertebral disc degeneration. J Cell Mol Med. 2018;22(1):261-76.

14. Wang $X$, et al. Mesenchymal stem cell-derived exosomes have altered microRNA profiles and induce osteogenic differentiation depending on the stage of differentiation. PLoS One. 2018;13(2):e0193059.

15. Crescitelli $R$, et al. Subpopulations of extracellular vesicles from human metastatic melanoma tissue identified by quantitative proteomics after optimized isolation. J Extracell Vesicles. 2020;9(1):1722433.

16. Minogue BM, et al. Characterization of the human nucleus pulposus cell phenotype and evaluation of novel marker gene expression to define adult stem cell differentiation. Arthritis Rheum. 2010:62(12):3695-705.

17. Chimal-Monroy J, et al. Analysis of the molecular cascade responsible for mesodermal limb chondrogenesis: Sox genes and BMP signaling. Dev Biol. 2003;257(2):292-301

18. Risbud MV, et al. Defining the phenotype of young healthy nucleus pulposus cells: recommendations of the Spine Research Interest Group at the 2014 annual ORS meeting. J Orthop Res. 2015;33(3):283-93.

19. Tang $X$, et al. Identifying molecular phenotype of nucleus pulposus cells in human intervertebral disc with aging and degeneration. J Orthop Res. 2016; 34(8):1316-26

20. Deng B, et al. Expression profiles of MMP-1 and TIMP-1 in lumbar intervertebral disc degeneration. Genet Mol Res. 2015;14(4):19080-6.

21. Risbud MV, et al. Stem cell regeneration of the nucleus pulposus. Spine J. 2004:4(6 Suppl):348S-53S.

22. Vonk LA, et al. Mesenchymal stromal/stem cell-derived extracellular vesicles promote human cartilage regeneration in vitro. Theranostics. 2018;8(4):90620.

23. Alcaraz MJ, Compan A, Guillen MI. Extracellular vesicles from mesenchymal stem cells as novel treatments for musculoskeletal diseases. Cells. 2019;9(1): 98. https://doi.org/10.3390/cells9010098.

24. Krock E, Rosenzweig DH, Haglund L. The inflammatory milieu of the degenerate disc: is mesenchymal stem cell-based therapy for intervertebral disc repair a feasible approach? Curr Stem Cell Res Ther. 2015;10(4):317-28.

25. Lu K, et al. Exosomes as potential alternatives to stem cell therapy for intervertebral disc degeneration: in-vitro study on exosomes in interaction of nucleus pulposus cells and bone marrow mesenchymal stem cells. Stem Cell Res Ther. 2017:8(1):108

26. Liao Z, et al. Exosomes from mesenchymal stem cells modulate endoplasmic reticulum stress to protect against nucleus pulposus cell death and ameliorate intervertebral disc degeneration in vivo. Theranostics. 2019; 9(14):4084-100

27. Adams MA, Roughley PJ. What is intervertebral disc degeneration, and what causes it? Spine. 2006:31(18):2151-61.

28. Bibby SR, et al. The pathophysiology of the intervertebral disc. Joint Bone Spine. 2001;68(6):537-42.

29. Xia C, et al. Mesenchymal stem cell-derived exosomes ameliorate intervertebral disc degeneration via anti-oxidant and anti-inflammatory effects. Free Radic Biol Med. 2019;143:1-15.

30. Hingert D, Barreto Henriksson $\mathrm{H}$, Brisby H. Human mesenchymal stem cells pre-treated with IL-1 beta and stimulated with BMP-3 enhance chondrogenesis. Tissue Eng Part A. 2018;24(9-10):775-85. https://doi.org/10. 1089/ten.TEA.2017.0087.

31. Hingert $D$, et al. Investigation of the effect of secreted factors from mesenchymal stem cells on disc cells from degenerated discs. Cells Tissues Organs. 2019;208(1-2):76-88. https://doi.org/10.1159/000506350.

32. Caplan Al, Correa D. The MSC: an injury drugstore. Cell Stem Cell. 2011;9(1): $11-5$. 
33. Tetta $\mathrm{C}$, et al. The role of microvesicles derived from mesenchymal stem cells in tissue regeneration; a dream for tendon repair? Muscles Ligaments Tendons J. 2012;2(3):212-21.

34. Wang $X$, et al. Exosomes influence the behavior of human mesenchymal stem cells on titanium surfaces. Biomaterials. 2020;230:119571.

35. Phinney DG, Pittenger MF. Concise review: MSC-derived exosomes for cellfree therapy. Stem Cells. 2017;35(4):851-8.

36. Strassburg S, et al. Bi-directional exchange of membrane components occurs during co-culture of mesenchymal stem cells and nucleus pulposus cells. PLoS One. 2012;7(3):e33739. https://doi.org/10.1371/journal.pone. 0033739.

37. Yanez-Mo M, et al. Biological properties of extracellular vesicles and their physiological functions. J Extracell Vesicles. 2015;4:27066. https://doi.org/10. 3402/jev.v4.27066. eCollection 2015.

38. Kandere-Grzybowska K, et al. IL-1 induces vesicular secretion of IL-6 without degranulation from human mast cells. J Immunol. 2003;171(9):4830-6.

39. Baj-Krzyworzeka M, et al. Tumour-derived microvesicles contain interleukin-8 and modulate production of chemokines by human monocytes. Anticancer Res. 2011;31(4):1329-35.

40. Truman $L A$, et al. CX3CL1/fractalkine is released from apoptotic lymphocytes to stimulate macrophage chemotaxis. Blood. 2008;112(13):5026-36.

\section{Publisher's Note}

Springer Nature remains neutral with regard to jurisdictional claims in published maps and institutional affiliations.

Ready to submit your research? Choose BMC and benefit from:

- fast, convenient online submission

- thorough peer review by experienced researchers in your field

- rapid publication on acceptance

- support for research data, including large and complex data types

- gold Open Access which fosters wider collaboration and increased citations

- maximum visibility for your research: over $100 \mathrm{M}$ website views per year

At BMC, research is always in progress.

Learn more biomedcentral.com/submissions 